\title{
In vivo microelectrode track reconstruction using magnetic resonance imaging
}

\author{
S.H. Fung ${ }^{\mathrm{a}, \mathrm{b}}$, D. Burstein ${ }^{\mathrm{a}, \mathrm{c}}$, R.T. Born ${ }^{\mathrm{b}, *}$ \\ a Harvard-MIT Division of Health Sciences and Technology, Harvard Medical School, Boston, MA 02115, USA \\ ${ }^{\mathrm{b}}$ Department of Neurobiology, Harvard Medical School, 220 Longwood Avenue, Boston, MA 02115, USA \\ ${ }^{\mathrm{c}}$ Department of Radiology and Charles A. Dana Research Institute, Beth Israel Deaconess Medical Center, and Harvard Medical School, Boston, \\ MA 02215, USA
}

Received 22 September 1997; received in revised form 5 December 1997; accepted 8 December 1997

\begin{abstract}
To obtain more precise anatomical information about cortical sites of microelectrode recording and microstimulation experiments in alert animals, we have developed a non-invasive, magnetic resonance imaging (MRI) technique for reconstructing microelectrode tracks. We made microelectrode penetrations in the brains of anesthetized rats and marked sites along them by depositing metal, presumably iron, with anodic monophasic or biphasic current from the tip of a stainless steel microelectrode. The metal deposits were clearly visible in the living animal as approximately $200 \mu \mathrm{m}$ wide hypointense punctate marks using gradient echo sequences in a 4.7T MRI scanner. We confirmed the MRI findings by comparing them directly to the postmortem histology in which the iron in the deposits could be rendered visible with a Prussian blue reaction. MRI-visible marks could be created using currents as low as $1 \mu \mathrm{A}$ (anodic) for $5 \mathrm{~s}$, and they remained stable in the brains of living rats for up to nine months. We were able to make marks using either direct current or biphasic current pulses. Biphasic pulses caused less tissue damage and were similar to those used by many laboratories for functional microstimulation studies in the brains of alert monkeys. (C) 1998 Elsevier Science B.V. All rights reserved.
\end{abstract}

Keywords: Functional architecture; Alert animals; Chronic electrophysiology; Microstimulation; Cortical mapping; Magnetic resonance imaging

\section{Introduction}

The ability to record neuronal signals from an animal that is alert and performing a task has greatly advanced our understanding of the physiological basis of perception and behavior (e.g. Newsome et al., 1989). Ideally, one would like to be able to determine the anatomic location of the recording sites during or immediately following the experiments. However, the currently available techniques for determining the electrode locations are, for the most part, postmortem techniques.

In this study we have developed an MRI technique

* Corresponding author. Tel.: + 1617 4321307; fax: + 1617 7347557; e-mail: rborn@warren.med.harvard.edu that exploits the high detectability of paramagnetic metals, such as iron, for visualizing the location of microelectrode recordings in vivo. The main goal of the work described here was to demonstrate the feasibility of the technique and to determine reasonable parameters for future MRI studies.

\section{Methods}

\subsection{Animals and surgery}

Experiments were performed on 12 adult Long-Evans rats using procedures approved by the Harvard Medical Area Standing Committee on Animals. 
For each experiment, a rat was anesthetized with sodium pentobarbital (35 mg/kg, i.p.). Its head was then shaved and placed in a small-animal stereotaxic frame (Narishige, Japan). The incisor bar was adjusted until the head was in the flat-skull position (approximately $3.3 \mathrm{~mm}$ below horizontal), thus allowing the animal's stereotaxic coordinates to be referenced to a standard rat brain atlas (Paxinos and Watson, 1986). With the head properly positioned, a midline incision was made to expose the frontal and parietal bones. With a high speed dental drill a small craniotomy was made in each hemisphere $3.5 \mathrm{~mm}$ lateral to the bregma to allow the insertion of a microelectrode.

Unless stated otherwise, stainless steel microelectrodes ( \# 21-10-1, SS304, Frederick Haer, Brunswick, ME) were used for making marks. Microelectrodes were $150 \mathrm{~mm}$ long and had shaft diameters of $0.25 \mathrm{~mm}$ before insulation (multiple coats of epoxylite), with impedances of $4.0 \pm 0.4 \mathrm{M} \Omega$ at the standard reference of $1 \mathrm{kHz}$. The tip profile was 'blunt', tapering from a shoulder diameter of $43-53 \mu \mathrm{m}$ to a point over the last $120 \mu \mathrm{m}$ of length.

The microelectrode tip was lowered into the brain either 3 or $4 \mathrm{~mm}$ lateral to the bregma using a microdrive (Narishige, Japan). Seven points were selected along each penetration, spaced at approximately 0.5 $\mathrm{mm}$ intervals, so that three marking sites were in the cortex and four were in the neostriatum. Either 1 or 2 penetrations were made in each hemisphere, all within the coronal plane containing the bregma, in order to facilitate imaging of the electrode marks.

The animals were allowed to recover, and MRI was done within 24 hours of surgery. Some animals were allowed to survive for longer periods post-surgery and were imaged at various time points (Table 1) after creation of the marks, as described below.

\subsection{Protocols for marking of tip locations}

Standard parameters. We first tried the minimum current parameter suggested by Green (1958) for identifying electrode recording sites by the Prussian blue method. Each site was marked with an anodic direct current pulse of $2 \mu \mathrm{A}$ for $15 \mathrm{~s}$ using a custom built constant current source. Eleven rats were marked.

\subsubsection{Stainless steel versus tungsten}

In order to determine if the MRI results were due to metal deposition or due to microlesions created by the passing current, we made parallel penetrations with the stainless steel microelectrodes described above and with tungsten microelectrodes (catalog \# 26-10-1, 99.9999\% tungsten, Frederick Haer, Brunswick, ME) using the same standard currents of $2 \mu \mathrm{A}$ for $15 \mathrm{~s}$. Three rats were used for this part of the study.

\subsubsection{Various amplitudes and durations of direct current pulse}

Once we established our imaging protocol, we tested the effects of varying the duration of the direct current pulse on the intensity and permanence of the marks seen with MRI. Currents of 1 or $2 \mu \mathrm{A}$ were used for $5-15 \mathrm{~s}$, and the brain images were made within $24 \mathrm{~h}$ of

Table 1

Summary of experiments

\begin{tabular}{|c|c|c|}
\hline $\begin{array}{l}\text { Animal identity } \\
\#^{\mathrm{a}}\end{array}$ & Electrode type ${ }^{\mathrm{b}}$ (parameters) & Imaging times \\
\hline SF07 & $\begin{array}{l}\text { SS (d.c. pulse, } 2.5 \mu \mathrm{A}, 2-5 \\
\text { s) }\end{array}$ & $<24 \mathrm{~h}$ \\
\hline SF09 & SS (d.c. pulse, $2 \mu \mathrm{A}, 15 \mathrm{~s}$ ) & $<24 \mathrm{~h}$ \\
\hline \multirow[t]{6}{*}{ SF12 } & $\begin{array}{l}\text { SS (d.c. current pulse, } 2 \mu \mathrm{A} \text {, } \\
15 \mathrm{~s} \text { ) }\end{array}$ & $<24 \mathrm{~h}$ \\
\hline & & 2 days \\
\hline & & 1 week \\
\hline & & 2 weeks \\
\hline & & 2 months \\
\hline & & 3 months \\
\hline SF13 & $\mathrm{W}$ (d.c. pulse, $2 \mu \mathrm{A}, 15 \mathrm{~s}$ ) & $<24 \mathrm{~h}$ \\
\hline & $\begin{array}{l}\text { vs. SS (d.c. pulse, } 2 \mu \mathrm{A}, 15 \\
\text { s) }\end{array}$ & \\
\hline SF14 & $\mathrm{W}$ (d.c. pulse, $2 \mu \mathrm{A}, 15 \mathrm{~s}$ ) & $<24 \mathrm{~h}$ \\
\hline & $\begin{array}{l}\text { vs. SS (d.c. pulse, } 2 \mu \mathrm{A}, 15 \\
\text { s) }\end{array}$ & \\
\hline $\mathrm{SF} 24^{\mathrm{c}}$ & $\mathrm{W}$ (d.c. pulse, $2 \mu \mathrm{A}, 15 \mathrm{~s}$ ) & $<24 \mathrm{~h}$ \\
\hline & $\begin{array}{l}\text { vs. SS (d.c. pulse, } 2 \mu \mathrm{A}, 15 \\
\text { s) }\end{array}$ & \\
\hline \multirow[t]{4}{*}{ SF15 } & SS (d.c. pulse, $1-2 \mu \mathrm{A}, 5-15$ & $<24 \mathrm{~h}$ \\
\hline & $\begin{array}{l}\text { s) vs. SS (d.c. pulse, } 2 \mu \mathrm{A} \text {, } \\
15 \mathrm{~s})\end{array}$ & \\
\hline & & 2 weeks \\
\hline & & 2 months \\
\hline SF17 & $\begin{array}{l}\text { SS (d.c. pulse, } 1-2 \mu \mathrm{A}, 5-15 \\
\text { s) vs. SS (d.c. pulse, } 2 \mu \mathrm{A} \text {, } \\
15 \text { s) }\end{array}$ & $<24 \mathrm{~h}$ \\
\hline \multirow[t]{5}{*}{$\mathrm{SF} 23^{\mathrm{d}}$} & SS (d.c. pulse, $1-2 \mu \mathrm{A}, 5-15$ & $<24 \mathrm{~h}$ \\
\hline & $\begin{array}{l}\text { s) vs. SS (d.c. pulse, } 2 \mu \mathrm{A}, 15 \\
\text { s) }\end{array}$ & \\
\hline & & 1 month \\
\hline & & 2 months \\
\hline & & 9 months \\
\hline SF16 & $\begin{array}{l}\text { SS (biphasic current pulse, } \\
10-60 \text { s) vs. SS (d.c. pulse, } 2 \\
\mu \mathrm{A}, 15 \mathrm{~s})\end{array}$ & $<24 \mathrm{~h}$ \\
\hline SF19 & $\begin{array}{l}\text { SS (biphasic current pulse, } \\
10-60 \text { s) vs. SS (d.c. pulse, } 2 \\
\mu \mathrm{A}, 15 \mathrm{~s})\end{array}$ & $<24 \mathrm{~h}$ \\
\hline \multirow[t]{3}{*}{$\mathrm{SF} 22^{\mathrm{e}}$} & $\begin{array}{l}\text { SS (biphasic current pulse, } \\
10-60 \text { s) vs. SS (d.c. pulse, } 2 \\
\mu \mathrm{A}, 15 \mathrm{~s})\end{array}$ & $<24 \mathrm{~h}$ \\
\hline & & 1 month \\
\hline & & 2 months \\
\hline
\end{tabular}

\footnotetext{
a These were all of the rats imaged.

${ }^{\mathrm{b}} \mathrm{SS}$, stainless steel; W, tungsten.

${ }^{\mathrm{c}}$ See Fig. 1.

${ }^{d}$ See Figs. 2 and 4 (C, D).

${ }^{\text {e }}$ See Figs. 3 and 4 (A, B).
} 
surgery and then at various subsequent time-points (Table 1). These marks were compared to those made in a parallel penetration using the standard current parameter of $2 \mu \mathrm{A}$ for $15 \mathrm{~s}$. Three rats were used for this study.

\subsubsection{Biphasic current pulse of various durations}

We also made penetrations in which we created marks using biphasic current pulses. These pulses were generated using a stimulus isolation unit connected to a biphasic pulse generator (BAK Electronics, Germantown, MD). Because of voltage compliance limitations of the stimulus isolation unit, we used microelectrodes of lower impedance $(<1 \mathrm{M} \Omega)$. The current pulses were biphasic, cathodal pulse leading, each pulse lasting 0.2 $\mathrm{ms}$ and separated by a gap of $0.1 \mathrm{~ms}$. The pulse pairs were $40 \mu \mathrm{A}$ in amplitude and delivered at a frequency of $200 \mathrm{~Hz}$. The duration of the pulse trains that we tested were $10,15,30$, and $60 \mathrm{~s}$. In all cases, the standard monophasic marks of $2 \mu \mathrm{A}$ for $15 \mathrm{~s}$ were created along parallel tracks for comparison. Three animals were used for this part of the study.

\subsection{Acquisition of MR images}

Imaging was performed using a Bruker Biospec 4.7 T, $30 \mathrm{~cm}$ bore MRI system (Bruker Instruments, Billerica, MA). For each imaging series, the animal was anesthetized with sodium pentobarbital $(35 \mathrm{mg} / \mathrm{kg}$, i.p.) and placed in a small, plastic sled to hold it securely in the bore of the magnet. A $7.5 \mathrm{~cm}$ slotted-tube radio frequency coil was used for imaging, with one exception (see Fig. 2D below). We first made a series of low resolution gradient echo scans (TR, $200 \mathrm{~ms}$; TE, $8 \mathrm{~ms}$; section thickness, $0.5 \mathrm{~mm}$; field of view (FOV), $3.8 \mathrm{~cm}$ over a matrix of $128 \times 128$; number of excitations (NEX), 4) in the sagittal plane in order to locate the craniotomy. This procedure took approximately 1-2 min of scanning time. The craniotomy as seen in the sagittal images was used to approximately center the next series (consisting of a packet of three sections) of low resolution scans in the coronal plane. This required another 1-2 min of scanning. If the marks were not seen on this series, the center point was moved either rostrally or caudally and a second low resolution coronal series was repeated. After finding the marks, we acquired a series of high resolution coronal gradient echo images centered on the site with a matrix of $256 \times 256, \mathrm{NEX}=30$, and other parameters identical. This series took an additional $25-30 \mathrm{~min}$, so that an entire imaging session lasted about $45 \mathrm{~min}$. For animals surviving longer than two weeks post-surgery, the craniotomy could no longer be detected on the low resolution sagittal scans due to healing of the bone. In these cases, the high resolution coronal images were centered using earlier sagittal scans in which the craniotomy could be located.
Because the image slices were not always parallel to the electrode penetrations, the metal deposit marks along each microelectrode track were often seen over two to three consecutive, non-overlapping MRI slices. A Matlab (MathWorks, Natick, MA) program was therefore written to reconstruct the microelectrode tracks and to display them within a single plane using a best-fit algorithm for determining the plane that contained the deposit marks as selected by the user.

\subsection{Histology}

At various time points after an electrode penetration and MR imaging, the animal was sacrificed with an overdose of sodium pentobarbital ( $>50 \mathrm{mg} / \mathrm{kg}$, i.p., to effect) and perfused through the heart with a ferrocyanide-containing fixative $(2.25 \%$ glutaraldehyde, $0.75 \%$ formaldehyde, $1 \%$ potassium ferrocyanide in 0.1 $\mathrm{M}$ phosphate buffer, $\mathrm{pH}$ 7.4). After fixation, the brain was removed from the skull and placed in $15 \%$ sucrose in $0.1 \mathrm{M}$ phosphate buffer at $4^{\circ} \mathrm{C}$ overnight or until it sank. The brain was then cut into $60 \mu \mathrm{m}$ coronal sections using a freezing sliding microtome. To demonstrate the iron within the metal deposits, we used the modified Prussian blue reaction procedure described by Brown and Tasaki (1961). The sections were wetted with $0.1 \mathrm{M}$ phosphate buffer and then treated with a solution of $2.5 \%$ ferricyanide, $2.5 \%$ ferrocyanide for 10 min. They were then transferred to a solution of $2.5 \%$ ferricyanide, $2.5 \%$ ferrocyanide, $10 \% \mathrm{HCl}$ for $30 \mathrm{~min}$. All reactions were carried out at room temperature. The sections were re-treated with $2.5 \%$ ferricyanide, $2.5 \%$ ferrocyanide for $10 \mathrm{~min}$ and then dehydrated in increasing concentrations of ethanol at 3 min intervals. The iron deposit marks were identified as azure spots (Figs. 1 and 4). Some of the sections were counterstained with neutral red to identify surrounding structures.

\section{Results}

\subsection{Standard parameters}

The standard current of $2 \mu \mathrm{A}$ (anodal) for $15 \mathrm{~s}$ through a stainless steel microelectrode consistently yielded punctate hypointense MRI-observable marks of approximately $200 \mu \mathrm{m}$ in diameter (Fig. 1). This was true of every attempt to image the metal deposits but two: in one case, rat \#SF12 at 2 weeks, there was a technical problem with the magnet (faulty filter diodes), and, in the second, rat \# SF12 at 3 months, we had not yet adopted an efficient search strategy prior to performing the high resolution scans. Subsequent to this case, the MR imaging was routine and extremely reliable. 

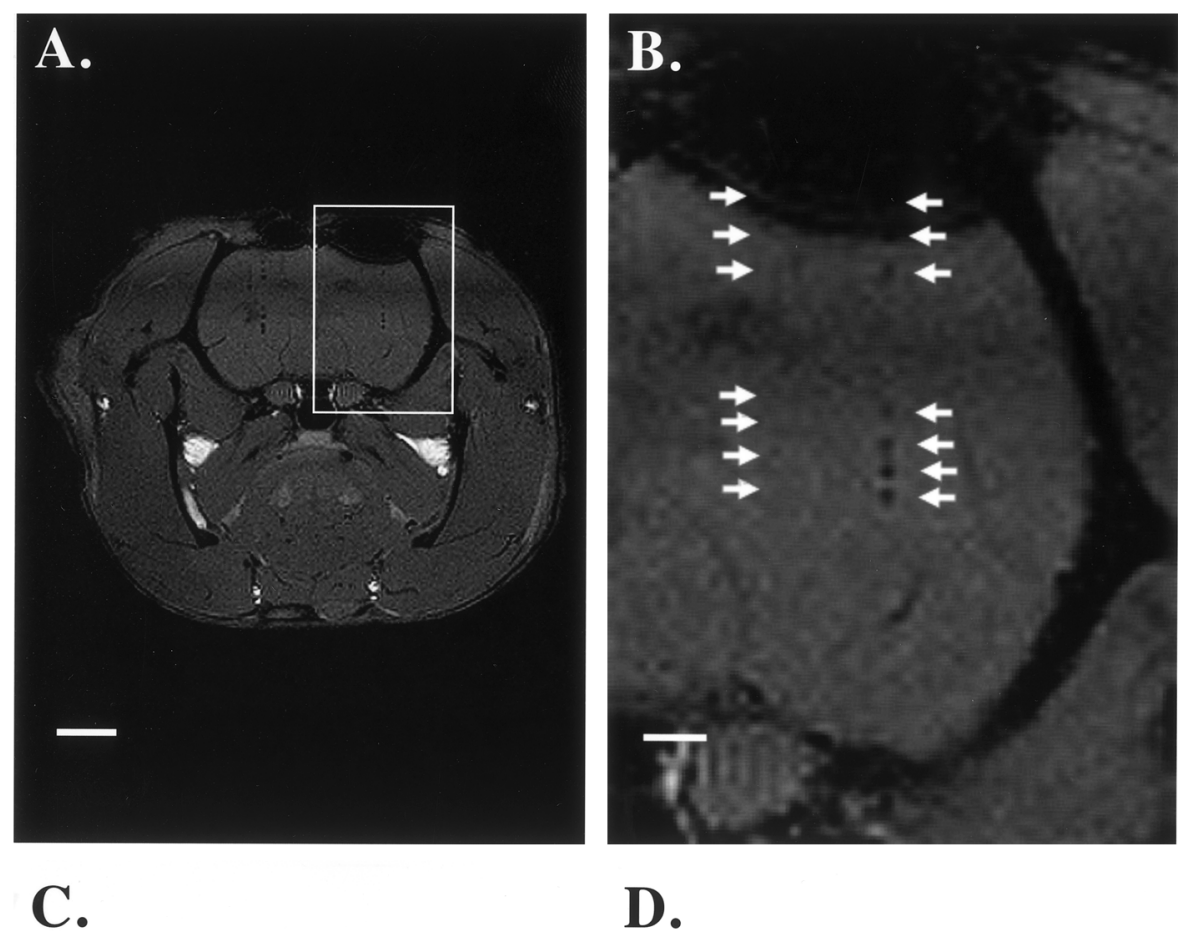

D.
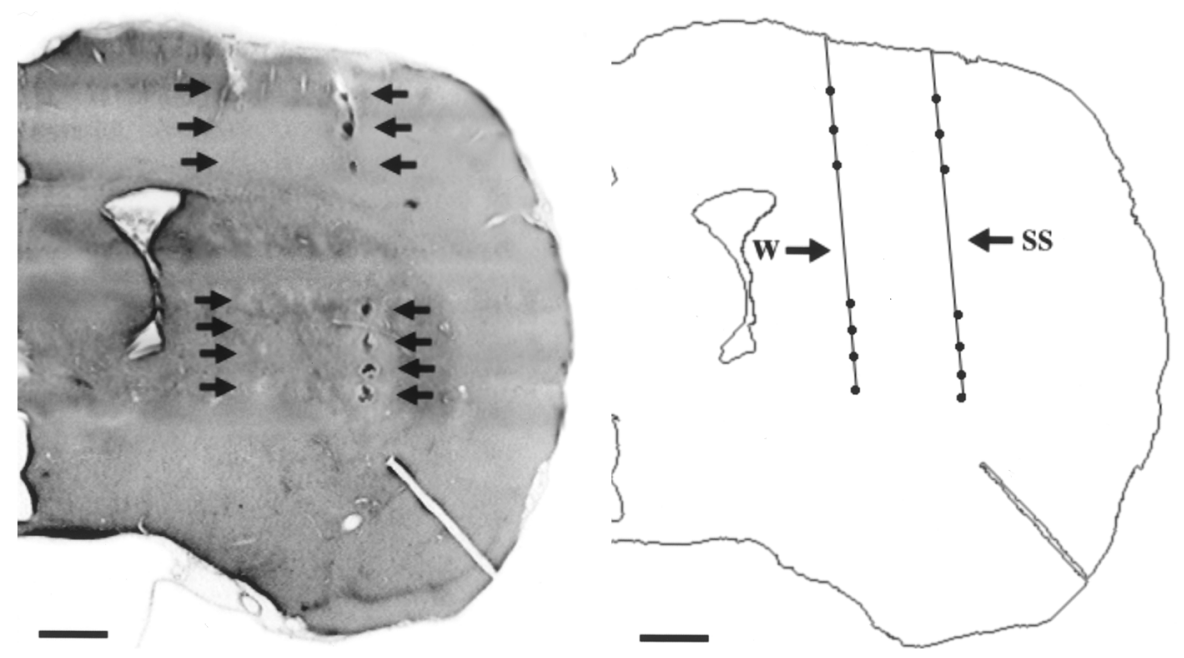

Fig. 1. Iron deposits visualized by MRI in a living, anesthetized rat. In each cerebral hemisphere of a rat (\# SF24), 2 electrode tracks were made, each with a total of 7 sites marked ( 3 in the cortex, 4 in the neostriatum). The leftmost track was made with a tungsten microelectrode, that on the right with a stainless steel microelectrode. The same current parameters were used at each site $(2 \mu \mathrm{A} \times 15 \mathrm{~s}$, anodal $)$. A, MR image obtained shortly after the marks were created. The animal's entire head is shown in coronal section, and two electrode tracks (one in each hemisphere) are just visible as vertical rows of dark dots. B, magnification of the rectangular region in A. Hypointense iron deposit marks are visible along only the rightmost (stainless steel) electrode track. The dorsal-most mark is not visible due to blood accumulation at the surface of the craniotomy. In this and all subsequent figures, the white arrows indicate locations at which current was passed-they do not imply that the marks were necessarily visible. C, histology from the same rat sacrificed immediately after obtaining the MR image in A and B. The iron in the deposits along the stainless steel track are visible (arrows) as a result of the Prussian blue reaction. Lesions created by passing current through the tungsten electrode were also visible in histological sections, but do not show up well on this photograph due to the lightness of the counterstain. D, schematic diagram of the electrode penetrations shown in B and C. Scale bars: $A=4 \mathrm{~mm}, \mathrm{~B}, \mathrm{C}=1 \mathrm{~mm}$.

\subsection{Stainless steel versus tungsten}

In order to confirm that it was the metal deposits we were imaging and not some other effect of passing current, we directly compared marking techniques using stainless steel and tungsten electrodes. Except for the metal composition, all parameters using the tungsten electrodes were identical to the ones used in the stainless steel marking technique. Although we could find the lesions created with the tungsten electrodes in the acute postmortem tissue sections, we could not detect them in our MR images (Fig. 1). The tungsten lesions 

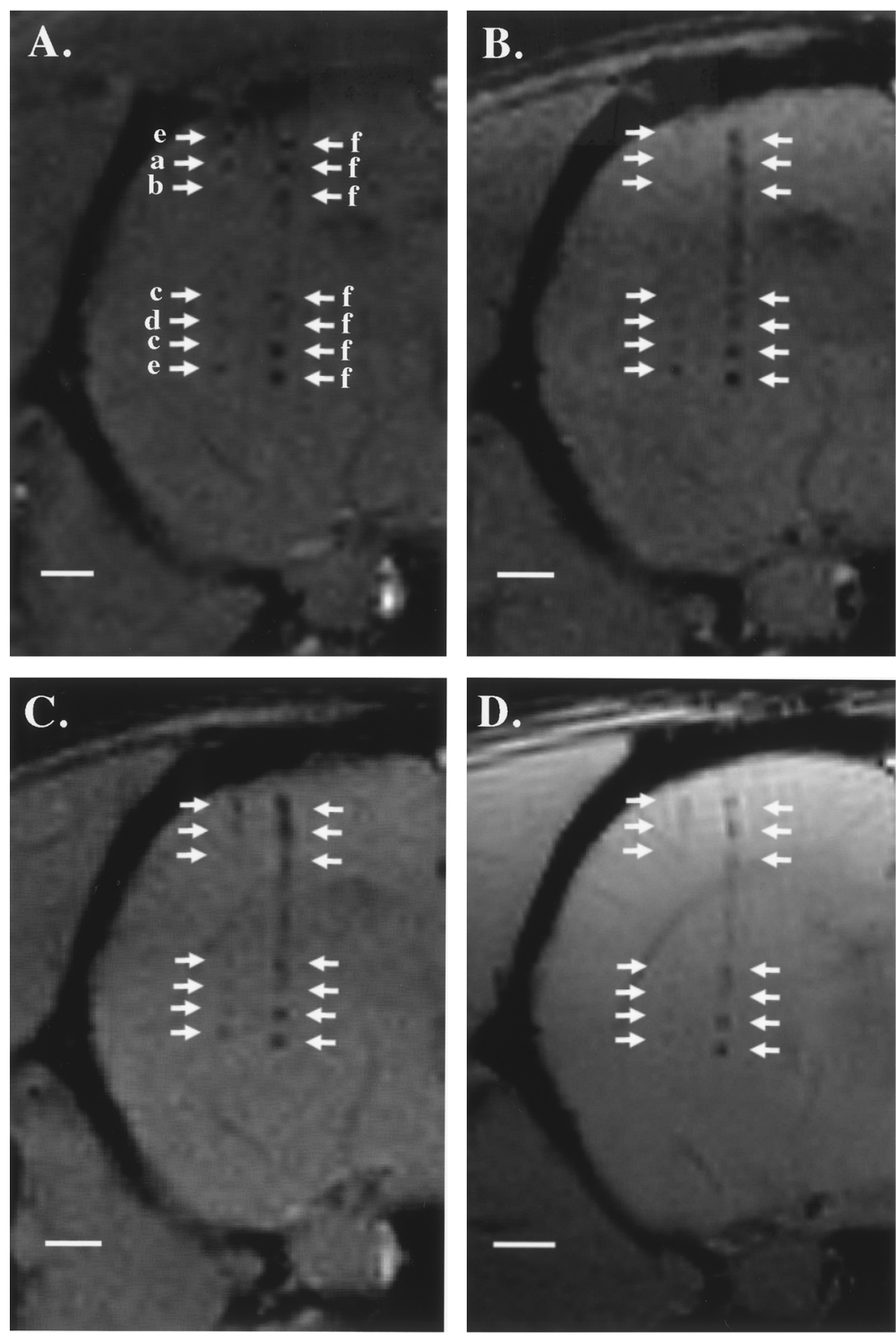

Fig. 2. Electrode tip marks produced by monophasic currents of varying amplitudes and durations. MR images obtained from a single animal (rat \# SF23) immediately after the electrode penetrations (A) and at one month (B), two months (C), and nine months (D) after the electrode penetrations. The iron deposits appear as dark spots. The image in panel $\mathrm{D}$ was obtained using a $2.5 \mathrm{~cm}$ receive only surface coil. The letters next to the marks in panel A indicate the current parameters used to make the marks: $\mathrm{a}=1 \mu \mathrm{A}$ for $5 \mathrm{~s}, \mathrm{~b}=1 \mu \mathrm{A}$ for $10 \mathrm{~s}, \mathrm{c}=1 \mu \mathrm{A}$ for $15 \mathrm{~s}, \mathrm{~d}=2$ $\mu \mathrm{A}$ for $5 \mathrm{~s}, \mathrm{e}=2 \mu \mathrm{A}$ for $10 \mathrm{~s}, \mathrm{f}=2 \mu \mathrm{A}$ for $15 \mathrm{~s}$. Scale bar $=1 \mathrm{~mm}$.

were hard to detect histologically after survival times greater than about one week, whereas the metal deposits remained visible with both MRI and histology for up to nine months (Figs. 2 and 4; see below). The visibility of the marks at two months was confirmed using MRI in four animals; that at nine months was done in a single animal. In another animal, imaged at three months, we failed to see any marks, but, as noted above, we believe that this was due to a problem with our search strategy and not a result of their disappearance.

\subsection{Varying amplitudes and durations of the direct current pulse}

Given that the marks created with our standard current parameters were easily seen and quite longlived, we were interested to know if passing lower currents for shorter times would also work. We were able to create MR-visible marks using currents as low as $1 \mu \mathrm{A}$ for $5 \mathrm{~s}$ (Fig. 2, mark 'a'), but these were fainter and less reliably detected, and they failed to last as long 

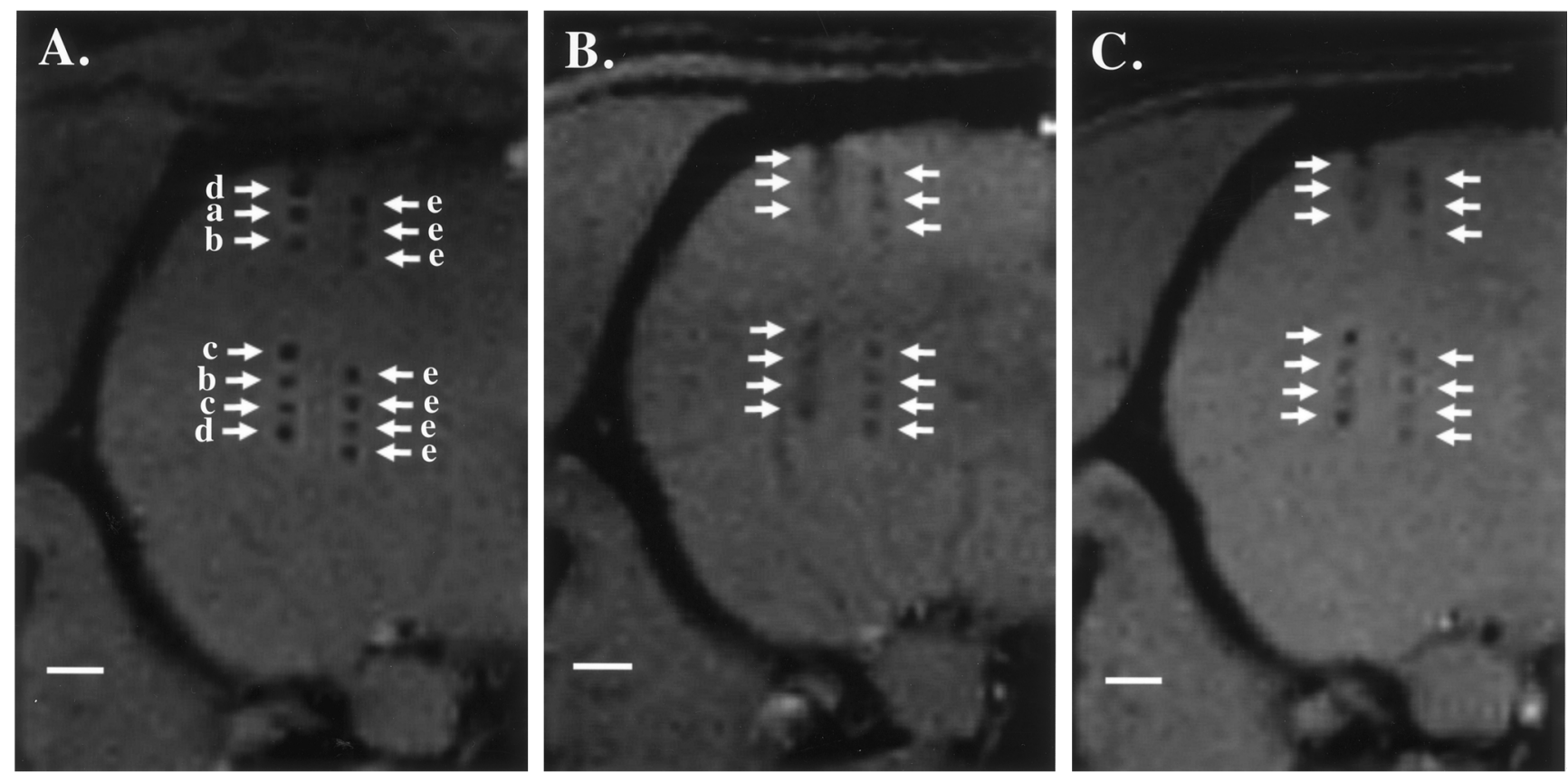

Fig. 3. Electrode tip marks produced by biphasic currents of varying durations. Comparison of MR marks made in a single animal (rat \# SF22) with biphasic (leftmost track in each figure) or monophasic (rightmost track) currents both immediately after the electrode penetration (A) and at one month (B) and two months (C) after the electrode penetration. Letters next to marks in panel A indicate the current parameters used to make the marks: a-d: biphasic ( $-40 \mu \mathrm{A}$ for $0.2 \mathrm{~ms}$, a $0.1 \mathrm{~ms}$ pause, followed by $+40 \mu \mathrm{A}$ for $0.2 \mathrm{~ms}$ at $200 \mathrm{~Hz})$ for various lengths of time: $\mathrm{a}=10, \mathrm{~b}=15, \mathrm{c}=30, \mathrm{~d}=60 \mathrm{~s}$; e: monophasic, $2 \mu \mathrm{A}$ for $15 \mathrm{~s}$. Scale bar $=1 \mathrm{~mm}$.

(Fig. 2B and C). The largest current we used $(2 \mu \mathrm{A}$ for 15 s) produced the most readily visible and longest lasting marks, and intermediate levels produced marks of intermediate visibility and permanence. While the sources of variance intrinsic to MR imaging (e.g. partial volume effect) precluded a meaningful parametric analysis, in all three rats used for this part of the study we observed a general proportionality between current parameters (amplitude and duration) and MRI results (detectability and permanence).

\subsection{Biphasic current pulse of varying durations}

Because microstimulation using trains of biphasic current pulses is a useful technique and because such trains cause much less tissue damage than direct current pulses (e.g. Salzman et al., 1992), we were also interested in knowing if we could make MRI-visible marks with these. Our goal was to simulate a microstimulation experiment at several sites along each electrode track. In an actual microstimulation experiment (e.g. Groh et al., 1997), the pulse train is turned on for only a subset of behavioral trials, and, in those trials, the pulse is on for only several hundred milliseconds. However, the net microstimulation time can be quite long, on the order of 50-100 s, since a typical experiment requires on the order of 1000 trials per site. As shown in Fig. 3, even short durations of microstimulation (10 and $15 \mathrm{~s}$ ) pro- duced marks that were clearly visible immediately after their creation but which faded over time, and by two months (Fig. 3C, a and b) became difficult to detect. The longer biphasic current pulse durations (30 and 60 s) produced marks that were still clearly visible even after a two month survival. This was confirmed in three animals.

\subsection{Effects on the microelectrodes}

After marking seven sites in each penetration, we observed, as expected, a decrease in the microelectrode's impedance and a blunting of its tip. For monophasic currents, the impedance of the microelectrode was generally lowered from approximately 4 to approximately $1.5 \mathrm{M} \Omega$ after making seven marks using $2 \mu \mathrm{A}$ for $15 \mathrm{~s}$ for each mark. This was also true for the biphasic currents. After making seven marks totaling $220 \mathrm{~s}$ of microstimulation time, the impedance of the microelectrode was generally lowered to around 0.3 $\mathrm{M} \Omega$ from the original approximately $0.9 \mathrm{M} \Omega$.

\subsection{Histology}

We performed postmortem histochemistry on each rat after its last imaging session to determine the relationship between the MR images and the histology. In general, detectability with the two methods (MRI ver- 
sus histochemistry) was similar (compare Fig. 1B and C). The histological material also gave us the opportunity to analyze the cellular location and nature of the metal deposits. Fig. 4 shows marks that were created two months (A and B) and nine months (C and D) before sacrificing the animal. At two months the high power image (Fig. 4B) shows marks consisting of a core of amorphous material surrounded by a ring of reactive gliosis. The mark's core had been invaded by phagocytic cells, probably microglia, many of which had internalized some of the iron-containing material. At even higher magnifications, it was seen that the phagocytized material was not uniformly distributed within the cell, but rather was accumulated in punctate bodies scattered throughout the cytoplasm. By nine months nearly all of the material was found within cells (Fig. 4D). A comparison of the histology at two and nine months showed some reduction in the size of the marks (compare Fig. 4A with Fig. 4C) with some tendency for iron deposit-containing microglia to migrate along the length of the electrode track (Fig. 4C). Even so, the original sites of metal deposition remained clearly identifiable.

\section{Discussion}

We have shown that MRI can be used to reconstruct electrode tracks reliably and at reasonably high spatial resolution in a living animal. Metal electrolytically deposited from the tip of a stainless steel electrode produced marks that were visible using MRI and Prussian blue histochemistry and were stable for up to nine months. The marks could be produced using either direct or biphasic current, the latter having the advantage of causing less tissue damage and of being a natural by-product of microstimulation experiments. We feel that the basic method of marking electrode tip location and performing MRI is straightforward and reliable enough to become a standard routine for any chronic physiology laboratory with access to an MRI scanner.We used a standard gradient echo sequence for imaging. In initial studies, a spin echo sequence was also found to be effective in detecting the marks; however, the signal to noise ratio was lower than that in the gradient echo scan for a given imaging time, as expected for spin echo scans in general.

Our failure to detect marks made with tungsten electrodes make it most likely that the basis of MRI detectability is a magnetic susceptibility artifact produced by the presence of paramagnetic ions (i.e. ions that possess unpaired electrons). The MR pulse sequences we used should be sensitive to the presence of any element with a high magnetic susceptibility. Of the elements present in SS304, this would include chromium, nickel and manganese as well as iron. On histological sections, the deep blue marks we saw were presumably due to the presence of iron, since the reaction products of other metals have different colors (Sharpe, 1976).

\subsection{Advantages and potential drawbacks}

The main advantage of the MRI technique is its suitability for in vivo imaging. Thus, microelectrode recording positions can be obtained immediately after the experiment's completion, and can be localized relative to anatomic markers.

There are a number of possible drawbacks to our method. The first is that, because the pulse sequences used to detect paramagnetic metals are, of necessity, very sensitive to metal-induced artifacts, standard stainless steel components for head fixation and cortical access should be avoided. This problem is easily circumvented using components made of a combination of high-strength plastic (Ultem, GE Plastics) and titanium, which are available commercially (Crist Instruments).

A second potential drawback to our method is the use of stainless steel electrodes, which are generally believed to be noisier than their tungsten or platinumiridium counterparts (see, for example, Snodderly and Gur, 1995). The electrodes we used were capable of recording single units (see Geddes et al., 1971 for a detailed treatment of the electrical properties of stainless steel electrodes). We did not do a detailed analysis of their recording characteristics, but we feel confident that they would be adequate for studies of functional organization and microstimulation experiments, where lower impedance electrodes are often desirable for recording multi-unit activity and for delivering currents within the compliance limits of commercially available stimulus isolation units.

Moreover, the use of stainless steel electrodes is not an absolute requirement of our technique - any electrode containing paramagnetic elements should work. Thus one might make electrodes from other alloys, such as elgiloy (Suzuki and Azuma, 1976), which are reported to have superior recording characteristics (Ashford et al., 1985). Alternatively, one might use Merrill's technique of iron plating on top of platinum black on a tungsten microelectrode (Merrill, 1974). Merrill reports that these electrodes "have all the desirable characteristics of plain glass-insulated tungsten electrodes" and that adding the layer of iron "does not increase the electrode's noise or impedance".

Two other drawbacks are related to the fact that current must be passed through the electrode in order to produce marks. This has the undesirable effects of altering the electrode's impedance (see last paragraph of results) and of creating microlesions which may affect physiological interactions. To a certain extent, 

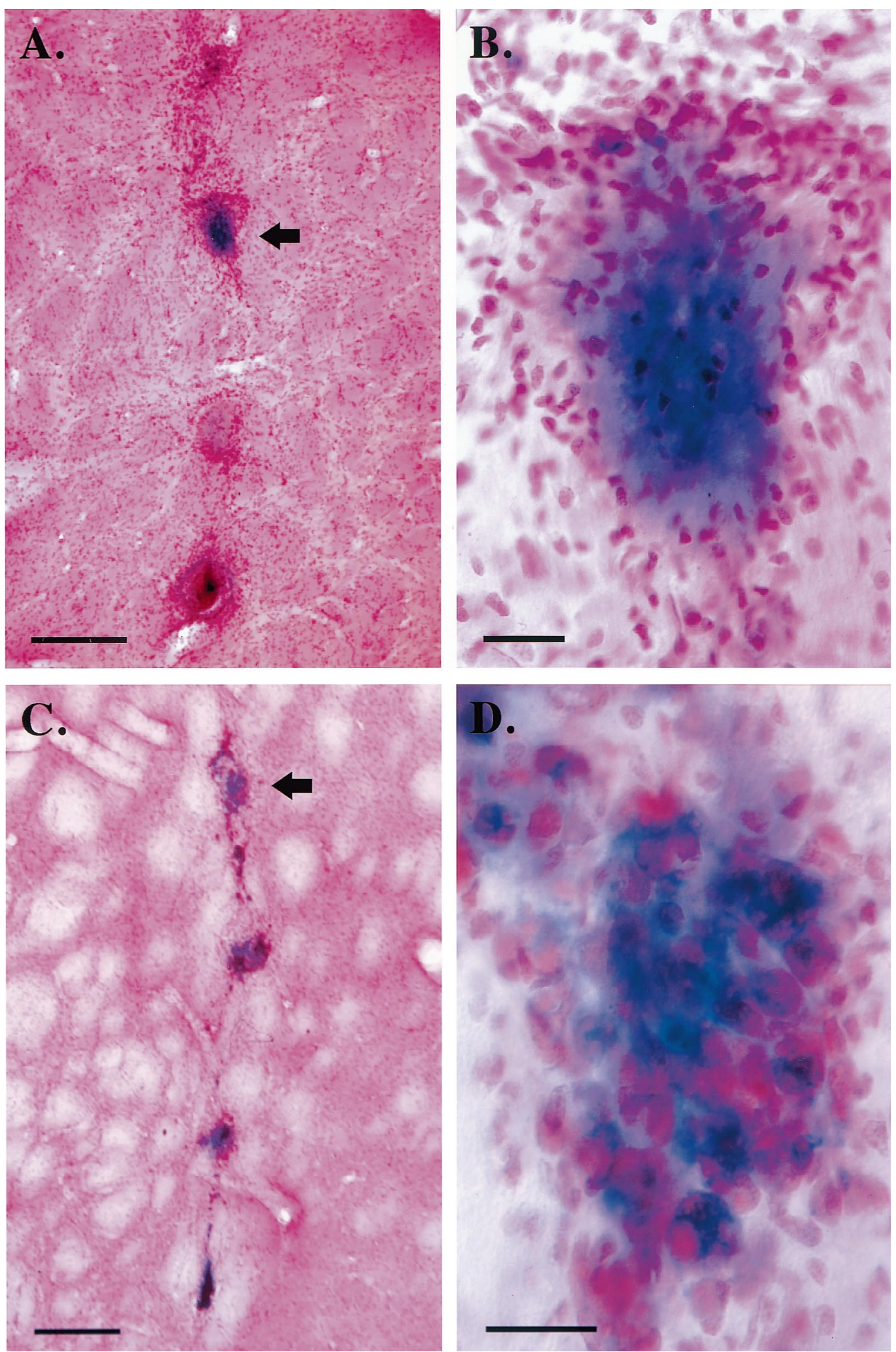

Fig. 4. Histological characterization of iron deposits. Electrode marks were created by passing monophasic, anodic current through a stainless steel microelectrode two months (rat \# SF22; A and B) or nine months (rat \# SF23; C and D) prior to sacrifice. The tissue was first processed for Prussian Blue histochemistry, then counterstained with neutral red. At low power (A, C), four blue marks are clearly visible in the neostriatum of each animal. The top mark in panel A is slightly out of the plane of section and so appears reduced in size. The second lesion from the top, magnified in panel B, shows several characteristics of the iron-containing marks. There is a central core of amorphous iron-containing material infiltrated by phagocytic cells and surrounded by a ring of reactive gliosis. By nine months (D), nearly all of the iron-containing material has been taken up by phagocytic cells, probably microglia. Scale bars: $A, C=200 \mu \mathrm{m} ; B, D=25 \mu \mathrm{m}$. 
both problems are unavoidable, but they can certainly be minimized by using smaller currents for shorter durations. The creation of microlesions can be further reduced, and perhaps eliminated, by using biphasic current pulses (Salzman et al., 1992).

Finally, if one wished to use larger currents so that the marks would persist for a longer time, there might be ambiguity between marks created at different sessions. One could potentially create a 'database' of MRI marks to differentiate new from old marks, or one could image with a grid system within the recording chamber, using an oil filled pipette to identify a given penetration on MRI.

Thus the MRI technique has the advantage of allowing the electrode recording site to be imaged in vivo. The technique can be optimized or tailored for use in specific applications. For example, in situations in which tissue damage is of particular concern and the marks do not need to be detectable for long periods, smaller current durations and/or biphasic currents can be used. Alternatively, not all recording sites need to be marked individually. Finally, if this technique is to be used extensively, specialized recording electrodes can be designed with the recording characteristics in mind but doped with paramagnetic substances to produce the MRI observable markings.

\subsection{Other methods}

Over the years, a number of methods for marking specific sites along microelectrode penetrations have been described. They include electrolytic lesions (Hubel, 1958; Hubel and Wiesel, 1962), depositing iron that is detected by the Prussian blue reaction (Hess, 1932; Adrian and Moruzzi, 1939; Marshall, 1940; Green, 1958; Brown and Tasaki, 1961), injecting dyes iontophoretically (Thomas and Wilson, 1965; Stretton and Kravitz, 1968; Lee et al., 1969), and coating the electrode with lipophilic fluorescent dyes (Honig and Hume, 1989; Snodderly and Gur, 1995; DiCarlo et al., 1996).

All of the above methods require sacrifice of the animal before anatomical information is obtained. There are fewer techniques available to the investigator who wishes to obtain such information while the animal is still alive. One solution has been the use of cortical biopsies (Hendry and Jones, 1988; Snodderly and Gur, 1995). This technique has the advantage of giving the high spatial resolution obtainable with histology, but it causes considerable local cortical damage which may alter the physiology of nearby neurons. Furthermore, the biopsy technique is not as feasible for recording sites located deep within the brain or for small cortical areas.

A second technique, developed by $\mathrm{Nahm}$ et al. (1994), uses a combination of MR and radiographic imaging to localize directly a metal microelectrode in the brain. It involves surgically attaching to the skull of the animal markers that are visible in both MRI and X-ray images. An MR database is then acquired before any metal hardware is affixed to the skull. Subsequent to this, standard chronic recording techniques (Evarts, 1968) are used and the position of a metal electrode is located using conventional X-rays. The known positions of the markers are then used to map the X-ray images of the electrode onto the more anatomically detailed MRI database. This method has the advantage of being compatible with standard stainless steel head hardware, but it is considerably more cumbersome than the method described here.

We see the methods outlined above as providing approaches complementary to the one we have developed. In certain cases, one or the other may be more practical, and even greater flexibility and spatial resolution might be obtained by using them in combination. For example, one might initially reconstruct the electrode tracks using an MR technique, and then proceed to do biopsies on sites that the MR images suggested were of particular interest.

\subsubsection{Possible uses of the technique}

This technique should be useful to investigators recording neuronal signals from alert animals, or employing microstimulation. With respect to anatomical localization of experimental sites, the current practice is to assign sites to a given cortical area by using a combination of functional criteria (i.e. single unit stimulus-response properties) and grid systems to map penetration coordinates onto postmortem histology. The accuracy of these methods is compromised by imprecision in the marking of sites from which one has recorded many days or months previously and by the lack of clear-cut markers to distinguish different cortical areas, even on the postmortem histology (Felleman and Van Essen, 1991). Using an MRI technique to reconstruct the electrode tracks can potentially solve both of these problems. It can eliminate the problem of long time intervals between the experiment and marking the experimental site: in the case of microstimulation, the mark is created as a by-product of the experiment, so there is no ambiguity in marking the experimental site; in the case of microelectrode recording, the marks can be made within minutes of the recordings using strategies similar to those used in acute experiments. The problem of distinguishing cortical areas is not yet eliminated by the MRI technique, but the ability to recover sites while an animal is still alive opens the possibility that areas can be defined using either functional MRI techniques, which have been used to delineate cortical visual areas in humans (Engel et al., 1994; Sereno et al., 1995), or by anatomical MRI techniques, which have the potential to allow the inves- 
tigator to identify cortical regions based on patterns of connectivity (Filler, 1994).

\section{Acknowledgements}

We are grateful for advice on histological techniques from Vladimir Berezovskii and for a thoughtful review of the manuscript by David Hubel, Margaret Livingstone, and Elio Raviola. James George provided valuable information on transition metal chemistry. This work was supported by NIH EY11379 (RTB) and a grant from the Milton Fund (RTB).

\section{References}

Adrian ED, Moruzzi G. Impulses in the pyramidal tract. J Physiol 1939;97:153-99.

Ashford JW, Coburn KL, Fuster JM. The elgiloy microelectrode: fabrication techniques and characteristics. J. Neurosci. Methods 1985; 247-52.

Brown KT, Tasaki K. Localization of electrical activity in the cat retina by an electrode marking method. J Physiol London 1961;158:281-95.

DiCarlo JJ, Lane JW, Hsiao SS, Johnson KO. Marking microelectrode penetrations with fluorescent dyes. J Neurosci Methods 1996;64:75-81.

Engel SA, Rumelhart DE, Wandell BA, et al. fMRI of human visual cortex. Nature 1994;369:525.

Evarts EV. A technique for recording activity of subcortical neurons in moving animals. Electroencephalog Clin Neurophysiol 1968;24:83-6.

Felleman DJ, Van Essen DC. Distributed hierarchical processing in the primate cerebral cortex. Cereb Cortex 1991;1:1-47.

Filler AG. Axonal transport and MR imaging: prospects for contrast agent development. J Magn Reson Imaging 1994;4:259-67.

Geddes LA, Da Costa CP, Wise G. The impedance of stainless-steel electrodes. Med Bio Eng 1971;9:511-21.

Green JD. A simple microelectrode for recording from the central nervous system. Nature 1958;182:962.

Groh JM, Born RT, Newsome WT. How is a sensory map read out? Effects of microstimulation in area MT on saccades and smooth pursuit eye movements. J Neurosci 1997;17:4312-30.
Hendry SH, Jones EG. Activity-dependent regulation of GABA expression in the visual cortex of adult monkeys. Neuron $1988 ; 1: 701-12$

Hess WR. Beitrage zur Physiologie des Hirnstammes. Leipzig: Thieme, 1932 .

Honig MG, Hume RI. DiI and DiO: versatile fluorescent dyes for neuronal labeling and pathway tracing. Trends Neurosci. 1989;12:333-41.

Hubel DH. Cortical unit responses to visual stimuli in nonanesthetized cats. Am J Ophthalmol 1958;46:110-22.

Hubel DH, Wiesel TN. Receptive fields, binocular interaction and functional architecture in the cat's visual cortex. J Physiol 1962;160:106-54.

Lee BB, Mandl G, Stean JP. Micro-electrode tip position marking in nervous tissue: a new dye method. Electroencephalog Clin Neurophysiol 1969;27:610-3.

Marshall WH. An application of the frozen sectioning technic for cutting serial sections thru the brain. Stain Technol 1940;15:1338.

Merrill EG. Iron-plated tungsten micro-electrodes for tip marking. Proc Physiolog Soc 1974;241:68P-9P.

Nahm FK, Dale AM, Albright TD, Amaral DG. In vivo microelectrode localization in the brain of the alert monkey: a combined radiographic and magnetic resonance imaging approach. Exp Brain Res 1994;98:401-11.

Newsome WT, Britten KH, Movshon JA. Neuronal correlates of a perceptual decision. Nature 1989;341:52-4.

Paxinos G, Watson C. The Rat Brain in Stereotaxic Coordinates, 2nd ed. San Diego, CA: Academic Press, 1986.

Salzman CD, Murasugi CM, Britten KH, Newsome WT. Microstimulation in visual area MT: effects on direction discrimination performance. J Neurosci 1992;12(6):2331-55.

Sereno MI, Dale AM, Reppas JB, et al. Borders of multiple visual areas in humans revealed by functional magnetic resonance imaging. Science 1995;268:889-93.

Sharpe AG. The Chemistry of Cyano Complexes of the Transition Metals. New York: Academic Press, 1976.

Snodderly DM, Gur M. Organization of striate cortex of alert, trained monkeys (macaca fascicularis): ongoing activity, stimulus selectivity, and widths of receptive field activating regions. J Neurophysiol 1995;74:2100-25.

Stretton A, Kravitz E. Neuronal geometry: determination with a technique of intracellular dye injection. Science 1968;162:132-4.

Suzuki H, Azuma M. A glass-insulated 'elgiloy' microelectrode for recording unit activity in chronic monkey experiments. Electroenceph Clin Neurophysiol 1976;41:93-5.

Thomas RC, Wilson VS. Precise localization of Renshaw cells with a new marking technique. Nature 1965;206:211-3. 